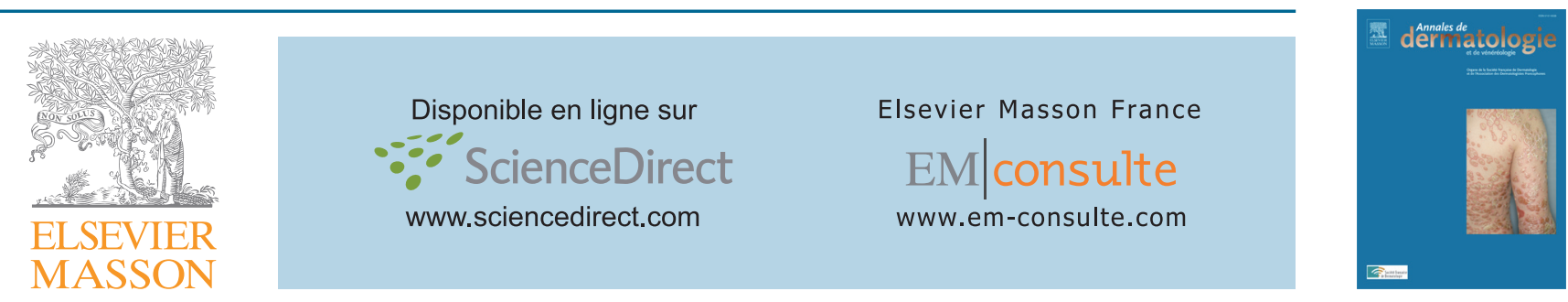

\title{
Acide Hyaluronique et matrice extracellulaire : une molécule primitive?
}

\section{Hyaluronic acid and extracellular matrix: a primitive molecule?}

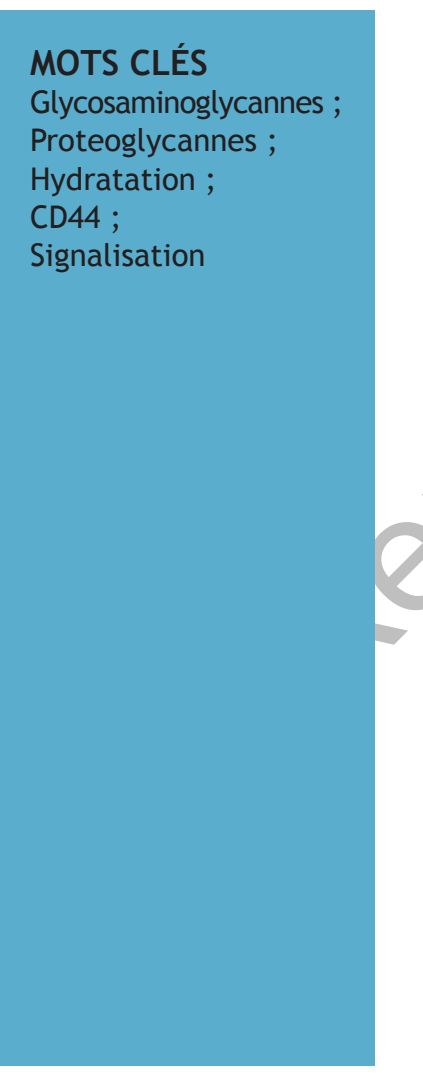

\section{B.-V. Nusgens}

Laboratoire de Biologie des Tissus Conjonctifs, GIGA-Recherche, Université de Liège, Belgique.

\begin{abstract}
Résumé
L'acide hyaluronique, ou hyaluronane, est un polymère constitué de la répétition d'une unité disaccharidique unique, acide $\mathrm{D}$-glucuronique et $\mathrm{D}-\mathrm{N}$-acétylglucosamine, dont la masse moléculaire peut atteindre 107 daltons. Cette structure primitive s'est révélée remarquable par ses propriétés viscoélastiques, ses capacités hygroscopiques et la diversité des processus cellulaires qu'elle contrôle. Identifié dans tous les tissus des vertébrés, plus de $50 \%$ de l'acide hyaluronique de l'organisme est présent dans la peau. Ne possédant pas de cœur protéique, sa synthèse procède d'un processus unique, dépendant de l'activité enzymatique d'hyaluronane synthases agissant à la face interne de la membrane plasmatique et extrudant le polymère en voie de formation vers le milieu extracellulaire. $\mathrm{Ce}$ polymère sert de matrice sur laquelle peuvent se fixer jusqu'à une centaine de chaînes de protéoglycannes sulfatés. Ces structures supramoléculaires de taille considérable peuvent entrapper de larges quantités d'eau et d'ions pour maintenir l'hydratation et la turgescence des tissus. L'acide hyaluronique est reconnu par la cellule grâce à la présence de récepteurs membranaires dont le mieux connu est CD44. L'interaction avec ses récepteurs active plusieurs cascades de signalisation intracellulaire régulant la prolifération, la migration et la différenciation. La réponse cellulaire est largement influencée par la taille du polymère d'acide hyaluronique qui se fixe au récepteur ou par celle des fragments générés par sa dégradation par les hyaluronidases et les radicaux libres. L'acide hyaluronique est métaboliquement extrêmement actif avec une demi-vie dans la peau de moins d'un jour. Détecté dans l'épiderme où il pourrait contrôler la prolifération et la différenciation des cellules basales, il est cependant majoritairement présent dans le derme en association avec le versican. Les propriétés physicochimiques remarquables de l'acide hyaluronique ainsi que la diversité des processus biologiques qu'il contrôle dépassent largement l'image primitive de ce polymère.
\end{abstract}

(c) 2010 Publié par Elsevier Masson SAS.

Auteur correspondant

Adresse e-mail : Betty.Nusgens@ulg.ac.be (B.-V. Nusgens) 


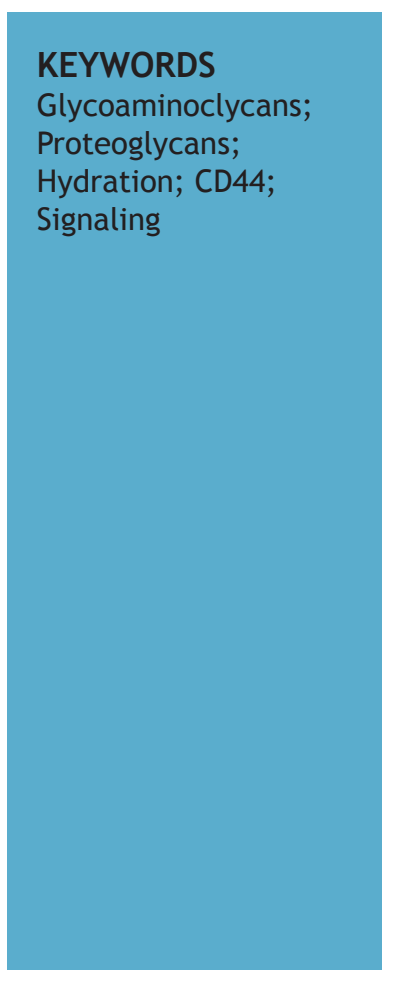

\begin{abstract}
Summary
Hyaluronic acid, or hyaluronan, is a polymer made of the repetition of a unique disaccharidic unit, D-glucuronic acid and D-N-acetylglucosamine, that can reach a molecular mass of $10^{7}$ daltons. This primitive polymer has emerged as a remarkable extracellular matrix component by its viscoelastic properties, its hygroscopic capacities and the diversity of cell processes it controls. Identified in all vertebrate tissues, more than $50 \%$ of acid hyaluronic of the organism is present in skin. Having no protein core, its synthesis is performed through a unique process, depending on enzymatic activity of hyaluronansynthases acting at the internal face of the plasmatic membrane and extruding the nascent polymer to the extracellular medium. This polymer constitutes a scaffold on which a large number of sulfated proteoglycans, up to one hundred, can be linked. These supramolecular structures of considerable size are able to entrap large amounts of water and ions to provide tissues with hydration and turgescence. Hyaluronic acid is recognized by cell membrane receptors, notably CD44 which is the best known. Interaction of hyaluronic acid with its receptors triggers several intracellular signaling pathways regulating proliferation, migration and differentiation. Cell response is largely influenced by the size of the polymer and by that of the fragments generated upon degradation by hyaluronidases or free radicals. Hyaluronic acid is metabolically very active since, for example, its half-life in skin is less than one day. Detected in epidermis where it could play a role in the control of proliferation and differentiation of basal cells, it is however prominent in dermis in association with versican. The remarkable physicochemical properties of hyaluronic acid as well as the diversity of biological processes it controls largely surpass the primitive character of this polymer.
\end{abstract} (c) 2010 Published by Elsevier Masson SAS.

Les composants de la matrice extracellulaire des tissus conjonctifs mous, bien qu'apparaissant en microscopie optique comme un matériel relativement amorphe remplissant l'espace intercellulaire, sont organisés en un réseau hautement structuré formé d'un échafaudage de protéines fibrillaires, collagène, élastine et microfibrilles en proportions variables, maintenu dans un gel visco-élastique et hydraté de glycosaminoglycannes, protéoglycannes et glycoprotéines. Ce réseau élaboré de macromolécules piège une grande quantité d'eau et d'ions, permet le flux des nutriments et des métabolites et comble les espaces interfibrillaires. La matrice extracellulaire, initialement considérée comme jouant essentiellement un rôle de support mécanique, s'est révélée au cours des dernières décennies détenir des propriétés informationnelles primordiales pour les cellules qu'elle supporte. La peau n'a pas échappé à cette progression des concepts. Parmi les composants prédominants de la matrice extracellulaire de la peau, l'acide hyaluronique y joue un rôle majeur en raison de ses propriétés rhéologiques, viscoélastiques et hygroscopiques remarquables. Il est apparu plus récemment que ce polymère exerce des activités biologiques sur les cellules non seulement par sa reconnaissance par des récepteurs membranaires et l'activation de voies de signalisation, mais également par sa translocation à l'intérieur de la cellule.

Les molécules d'acide hyaluronique sont capables d'interagir entre elles pour former des polymères de grande taille et peuvent également s'associer à d'autres molécules, les hyaladhérines, pour former une variété de structures impliquées dans le contrôle de la motilité, la prolifération, l'adhésion et l'activation de nombreux types cellulaires. L'acide hyaluronique est particulièrement abondant dans les tissus à renouvellement rapide, au cours du développement, de la cicatrisation tissulaire et dans certaines situations pathologiques, comme l'inflammation et la progression tumorale [1, 2].

\section{Un peu d'histoire}

Le terme « ground substance » que l'on pourrait traduire par «substance fondamentale » a été utilisé au début du XXème siècle pour décrire le matériel amorphe déposé au sein de l'espace intercellulaire des tissus conjonctifs mous. En identifiant quelques décennies plus tard l'hyaluronidase comme étant la substance active d'un extrait testiculaire capable de dissoudre cette substance fondamentale et faciliter la diffusion de substances injectées en sous-cutané («spreading factor») ou accroître l'invasivité bactérienne, la nature polysaccharidique des composants de la substance fondamentale était établie et supportait la dénomination alternative de «mucopolysaccharide ». C'est Karl Meyer, au siècle dernier, dans les années 30 , qui a démontré que l'acide hyaluronique était un constituant majeur de la substance fondamentale de l'humeur vitrée de l'œil responsable de sa turgescence [3]. Le terme d'acide hyaluronique est basé sur cette observation et provient du grec « hyalos» (vitreux) et uronique en raison de la présence d'acide hexuronique. Sa dernière appellation « hyaluronane » date de 1984 et se conforme à la nomenclature internationale des polysaccharides. Sa structure chimique a été élucidée dans le laboratoire de Karl Meyer au début des années 50 . Ce polymère est étonnant par la monotonie de sa structure puisqu'il est constitué de milliers de répétitions d'une seule et même unité disaccharidique. Cette structure primitive s'est pourtant révélée remarquable par ses propriétés physicochimiques et par la diversité des processus cellulaires qu'elle contrôle. 


\section{Structure chimique et physique de l'acide hyaluronique}

L'acide hyaluronique est un polymère linéaire formé d'unités disaccharidiques répétitives d'acide D-glucuronique et de $\mathrm{D}$ - $\mathrm{N}$-acétylglucosamine, liées entre-elles par des liaisons glycosidiques alternées $\beta-1,4$ et $\beta-1,3$. Ce polymère possède une masse moléculaire qui peut varier de $10^{5}$ à $10^{7}$ Daltons et s'étendre sur une longueur de 2 à $25 \mu \mathrm{m}$. Contrairement aux autres glycosaminoglycannes, l'acide hyaluronique n'est jamais sulfaté et n'est pas associé à un cœur protéique. Sa structure chimique lui confère un caractère très anionique qui lui permet de s'associer à de nombreux composants au travers d'interactions électrostatiques robustes. D'après les études physicochimiques, l'acide hyaluronique apparaît comme un polymère linéaire relativement rigide, en raison de liaisons hydrogènes entre les sucres adjacents et d'une répulsion mutuelle entre les résidus carboxyliques. Il présente une configuration hélicoïdale expansée pouvant retenir des quantités d'eau considérables. II peut cependant adopter des configurations variables en fonction du $\mathrm{pH}$, de la concentration en sels et des cations associés. Même à faible concentration, les solutions d'acide hyaluronique présentent une viscosité élevée. A plus haute concentration, cette viscosité s'accroît dramatiquement car les polymères linéaires sont capables de s'auto-associer et de s'enchevêtrer pour former des structures supramoléculaires gigantesques stabilisées par des liaisons hydrophobes intercaténaires [4]. Sa viscoélasticité lui permet de s'infiltrer facilement dans les tissus, de distendre et occuper des volumes significatifs de l'espace extracellulaire. Par encombrement stérique, il peut exclure d'autres macromolécules et retarder la diffusion de substances ou la migration de cellules dans le tissu. C'est, de ce fait, un excellent biomatériau de comblement qui peut subir des déformations tout en entrappant l'eau et les ions nécessaires au maintien de l'hydratation tissulaire. Ces polymères peuvent donc agir en tant que lubrifiant comme dans le liquide synovial et d'amortisseur en absorbant les chocs et charges de compression comme dans le cartilage. Il est également un matériel de comblement comme dans l'humeur vitrée de l'œil et la gelée de Wharton du cordon ombilical. L'acide hyaluronique est la première substance extracellulaire élaborée au cours des premiers stades du développement embryonnaire et forme une matrice hydratée autour du blastocyste. Il est également un composant important de la niche des cellules souches en fournissant un environnement propice au maintien de leur caractère pluripotent, en prévenant leur différenciation et en créant des voies sur lesquelles migrent les cellules souches durant le développement embryonnaire [5]. Il est particulièrement abondant au cours des processus de régénération et de réparation tissulaire. Cette molécule primitive a été identifiée dans tous les tissus des vertébrés, à des concentrations élevées dans l'humeur vitrée, le liquide synovial et le cordon ombilical. Il est intéressant de noter que plus de $50 \%$ de la totalité de l'acide hyaluronique de l'organisme $(20 \mathrm{~g})$ se trouve dans la peau.

\section{Le mécanisme de synthèse de l'acide hyaluronique est unique}

L'acide hyaluronique est donc une molécule purement saccharidique ne possédant pas de fraction protéique et dont la synthèse procède d'une façon inhabituelle. Alors que les autres glycosaminoglycannes sont attachés covalentiellement à une chaîne protéique dans l'appareil de Golgi, l'acide hyaluronique est synthétisé de façon tout à fait unique à la face interne de la membrane cellulaire, le polymère naissant étant extrudé au travers de la membrane vers l'extérieur au fur et à mesure qu'il s'allonge par addition alternée d'un acide glucuronique et d'une unité $\mathrm{N}$-acétylglucosamine. $\mathrm{Ce}$ mode de synthèse permet donc une croissance non limitée du polymère qui ne pourrait se dérouler dans le Golgi ou le réticulum endoplasmique sans détruire la cellule en raison de sa taille. Il n'y a guère qu'un peu plus de 10 ans qu'une famille multigénique d'enzymes responsables de la synthèse d'acide hyaluronique, les hyaluronanesynthases (HAS), a été découverte. La première HAS a été clonée chez une bactérie pathogène qui accroît sa virulence en s'entourant d'une capsule d'acide hyaluronique. Plusieurs groupes ont ensuite réussi à cloner et caractériser chez les mammifères trois gènes distincts ayant une activité HAS. Les trois HAS se distinguent par leur expression temporelle au cours du développement, par leur activité spécifique et par la taille des polymères d'acide hyaluronique qu'elles génèrent. L'HAS 2 semble jouer un rôle-clé au cours du développement puisque la délétion de son gène chez la souris est létale à mi-gestation en raison de nombreuses malformations cardiovasculaires. L'identification des enzymes opérant la synthèse de l'acide hyaluronique et leur mode d'activité a fait faire un bond significatif dans la compréhension de la biologie de l'acide hyaluronique et de sa régulation. L'acide hyaluronique est synthétisé par les cellules mésenchymateuses, épithéliales et immunitaires ainsi que par les cellules souche mésenchymateuses et hématopoiétiques [6].

La présence d'un gène codant pour une HAS dans diverses bactéries a permis la production d'acide hyaluronique à grande échelle par fermentation, ce qui représente une avancée significative par rapport aux modes de fabrication antérieurs. En effet, l'extraction d'acide hyaluronique à partir de tissus, comme la crête de coq, et sa purification pour éliminer toutes les protéines contaminantes nécessitaient l'application de procédés longs et coûteux. Il est intéressant de noter que phylogénétiquement, il existe une similarité de séquence frappante entre les HAS des vertébrés et les synthases des plantes et des champignons qui synthétisent, respectivement, la cellulose et la chitine, deux autres polymères qui avec l'acide hyaluronique sont les seuls à contenir des liaisons glycosidiques de type $\beta$.

\section{L'acide hyaluronique et ses partenaires : les hyaladhérines et les autres}

Les propriétés viscoélastiques uniques de l'acide hyaluronique sont certainement essentielles à ses fonctions biologiques. La 
compréhension de ses propriétés biologiques s'est cependant fortement élargie lorsque il fut montré dans les années 70 que ce polymère servait de matrice sur laquelle pouvait se fixer un grand nombre de molécules de protéoglycannes appartenant à la superfamille des « hyaladhérines», molécules capables d'interagir avec l'acide hyaluronique. Ces protéoglycannes, également dénommés hyalectans, contiennent en amino-terminal un domaine G1 (« module de liaison ») très conservé permettant leur fixation à l'acide hyaluronique, interaction stabilisée par une petite protéine de liaison agrafant solidement les deux molécules entre elles. Le rôle essentiel joué par ces protéines de liaison est démontré par la létalité précoce des souris KO pour le gène codant pour la protéine de liaison stabilisant le complexe acide hyaluronique-aggrécan. La famille des hyalectans comprend quatre membres : le versican, exprimé par plusieurs types de cellules mésenchymateuses chez l'adulte dont les fibroblastes et les cellules musculaires lisses, l'aggrécan surtout présent dans le cartilage, le neurocan et le brévican, deux protéoglycannes présents dans le système nerveux central.

Un polymère d'acide hyaluronique, pouvant atteindre une masse moléculaire de plusieurs millions de $\mathrm{Da}$, sur lequel se fixent de nombreuses molécules d'hyalectans, chacune composée d'une chaîne protéique de 100 à 370 kDa portant de 3 à 100 extensions de glycosaminoglycannes sulfatés, représente une structure de taille considérable. En s'imbriquant dans le réseau de fibres et de faisceaux de collagène, ces structures supramoléculaires déterminent la forme et l'organisation architecturale des tissus et leur confèrent des propriétés mécaniques appropriées à leurs fonctions tout en entrappant de larges quantités d'eau et d'ions pour maintenir leur hydratation et leur turgescence. Ces complexes sont impliqués par exemple dans la fonction d'amortisseur des cartilages, participent à l'élasticité des vaisseaux sanguins et contribuent au maintien de l'intégrité et l'hydratation de nombreux tissus comme la peau et le cerveau. Si l'on imagine que la nature, le nombre, la taille et la sulfation des extensions des glycosaminoglycannes peuvent être éminemment variables et que la chaîne protéique peut de surcroît exister sous plusieurs formes d'épissage alternatif, nous sommes confrontés à une myriade de molécules pouvant détenir des propriétés biologiques subtilement ou franchement différentes. Outre les hyaladhérines extracellulaires décrites ci-dessus, il existe un certain nombre de protéines associées à la surface des cellules et fixant l'acide hyaluronique à leur périmètre. Ces hyaladhérines cellulaires sont variées à la fois dans leur expression tissulaire, localisation cellulaire, spécificité, affinité et régulation, ajoutant un niveau de complexité à la compréhension de la fonction biologique de l'acide hyaluronique. Certaines hyaladhérines cellulaires ont en commun une séquence similaire au module de liaison des hyalectans et des protéines de liaison. C'est le cas de CD44, le récepteur le mieux connu et largement ubiquitaire de l'acide hyaluronique. Cette glycoprotéine transmembranaire peut s'exprimer sous de nombreuses isoformes produites par épissage alternatif d'un gène unique. Il subit par ailleurs des modifications post-traductionnelles qui élargissent encore la variabilité de fonctions de cette molécule dont beaucoup d'aspects restent à découvrir. La liaison entre l'acide hyaluronique et le CD44 peut activer plusieurs cascades de signalisations régulant la prolifération cellulaire et la migration [7]. Ces cascades de signalisations impliquent notamment les petites RhoGTPases RhoA et Rac1 qui interviennent dans l'organisation architecturale du cytosquelette et la migration, erbB2 et les MAPkinases contrôlant la prolifération, les tyrosine-kinases de la famille src et NFкB. Outre la complexité de la signalisation de CD44 liée à ses nombreuses isoformes, la taille des polymères d'acide hyaluronique qu'il fixe, influence également la réponse cellulaire. C'est ainsi qu'un polymère d'acide hyaluronique de grande taille est antiangiogène alors que des fragments de plus petite taille sont proangiogènes, attirent et activent les cellules inflammatoires [8]. Plus récemment, un récepteur à l'acide hyaluronique, LYVE-1, présentant $40 \%$ d'homologie avec CD44, a été mis en évidence dans les cellules endothéliales des vaisseaux lymphatiques [9]. Ce récepteur spécifique semble être impliqué dans le transport lymphatique de l'acide hyaluronique et sa dégradation. D'autres hyaladhérines capables de fixer l'acide hyaluronique par l'intermédiaire de courtes séquences d'acides aminés basiques ont été identifiées. Parmi celles-ci, RHAMM est un récepteur présent à la fois à la surface cellulaire, dans le cytoplasme et dans le noyau. Sa liaison avec l'acide hyaluronique active également de nombreuses voies de signalisation et est impliquée dans la promotion d'un phénotype migratoire et invasif, notamment dans les cellules tumorales. Les récepteurs à l'acide hyaluronique peuvent également, outre un rôle de signalisation, retenir l'acide hyaluronique à la surface cellulaire pour former une matrice péricellulaire volumineuse, encore appelée « glycocalyx ». Cette matrice péricellulaire excède souvent $20 \mu \mathrm{m}$ ce qui correspond plus ou moins à la longueur d'un polymère d'acide hyaluronique maintenu sous une conformation complètement étirée par association avec des hyalectans, aggrécan dans le cas des chondrocytes et versican dans le cas des fibroblastes et cellules musculaires lisses. Les HAS peuvent également retenir les polymères d'acide hyaluronique en voie de synthèse à la surface cellulaire Cette matrice péricellulaire hautement dynamique joue de multiples rôles [10]. Elle a été impliquée dans l'assemblage des macromolécules de la matrice extracellulaire, collagène et fibronectine, dans le contrôle de la prolifération et le phénotype migratoire de certaines cellules activées par des facteurs de croissance, comme le PDGF sur les cellules musculaires lisses. La différenciation du fibroblaste en myofibroblaste induite par TGF- $\beta 1$ est associée à une accumulation d'acide hyaluronique autour de la cellule. Par ailleurs, des études montrent que l'acide hyaluronique dans ces structures péricellulaires peut avoir soit un effet anti-adhésif ou pro-adhésif. Par exemple, il favoriserait le détachement cellulaire avant la mitose et fournirait une matrice anti-adhésive éminemment dynamique aux cellules en mouvement, notamment les kératinocytes de l'épiderme [11]. Par contre, l'acide hyaluronique est clairement proadhésif lorsqu'il est projeté par la cellule sous la forme de câbles qui induisent l'adhésion des monocytes dans un environnement inflammatoire, in vitro et in vivo. Enfin, étant donné que les récepteurs de l'acide hyaluronique interagissent avec le cytosquelette, la matrice péricellulaire serait impliquée dans la mécanotransduction et la réponse cellulaire aux variations de tension mécanique. 


\section{Dégradation de l'acide hyaluronique et vitesse de renouvellement}

L'acide hyaluronique est métaboliquement extrêmement actif puisque sa demi-vie n'est que de 3 à 5 minutes dans la circulation, moins d'un jour dans la peau et même dans des tissus plus inertes comme le cartilage, elle est de 1 à 3 semaines, ce qui est très rapide par comparaison aux protéines fibrillaires comme le collagène et l'élastine. Cette vitesse de renouvellement extraordinairement rapide implique que ce polymère est constamment synthétisé et constamment dégradé. La dégradation de l'acide hyaluronique est réalisée en première instance par des hyaluronidases («spreading factor ») qui opèrent comme des enzymes endoglycolytiques en clivant la liaison $\beta 1$-4 et génèrent des oligosaccharides de taille variable. Cette première cassure endolytique est suivie par le clivage des sucres. Il existe dans le génome humain six gènes codant pour des hyaluronidases de distribution tissulaire variée [12]. Par ailleurs, l'acide hyaluronique polymérique peut être dégradé de manière non-enzymatique par les radicaux libres et constitue un piège pour ces ions réactionnels. La dégradation progressive des polymères de grande taille de l'acide hyaluronique par une succession de réactions enzymatiques génère des polymères de taille décroissante qui exercent des fonctions biologiques extrêmement variées et souvent opposées [13]. Les mécanismes sous-tendant cette signalisation opposée sont encore mal définis mais pourraient impliquer une compétion pour les récepteurs membranaires et induire des réponses inflammatoires, immuno-stimulantes et angiogéniques.

Le turn-over rapide de l'acide hyaluronique est dû, en partie, à son drainage à partir des tissus d'où il est produit vers les vaisseaux lymphatiques où environ $85 \%$ est dégradé. Les $15 \%$ restants qui atteignent le torrent circulatoire sont rapidement éliminés, avec une demi-vie de 3 à 5 minutes, au niveau du foie et des reins. Le métabolisme intense de l'acide hyaluronique est réminiscent des processus de survie en réponse à un stress. La capacité de pouvoir fournir immédiatement de grandes quantités d'acide hyaluronique en réponse à un stress aigu, comme un choc, une hémorragie ou une septicémie est vraisemblablement liée à une réduction de sa dégradation plutôt qu'à une activation de sa synthèse, un processus plus long et moins efficace à mettre en place.

\section{Acide hyaluronique et peau}

L'acide hyaluronique est présent dans pratiquement tous les tissus et fluides des organismes vertébrés mais la peau en est le réservoir le plus important puisqu'elle contient $50 \%$ de la quantité totale de l'organisme. Les études histologiques ont vraisemblablement largement sous-estimé la présence d'acide hyaluronique dans la peau en raison de sa solubilité dans les fixateurs aqueux comme la formaline, surtout au niveau de l'épiderme. Une acidification et l'addition d'éthanol au fixateur est requise afin d'éviter la disparition de l'acide hyaluronique. Grâce à l'utilisation de peptides marqués se liant à l'acide hyaluronique et les modèles de peau reconstituée, il a pu être montré que l'épiderme contenait de l'acide hyaluronique, démontrant par là que les cellules mésenchymateuses du derme ne sont pas les seules à le produire. Les kératinocytes utilisent majoritairement la HAS-3 et en moindre mesure la HAS-2 pour produire l'acide hyaluronique, au moins in vitro. Il est retrouvé surtout au niveau des couches spineuses et granuleuses de l'épiderme, principalement dans l'espace intercellulaire, alors qu'il est présent dans la couche basale mais en localisation intracellulaire. Il pourrait y jouer un rôle dans le contrôle de la prolifération cellulaire et de la différenciation des cellules basales. Il serait associé à des mécanismes de dissociation cellulaire dans les couches supérieures. En culture, lorsque la concentration de calcium est basse, les kératinocytes produisent des quantités importantes d'acide hyaluronique et prolifèrent rapidement. En accroissant la concentration en calcium dans le milieu de culture, les kératinocytes entament un programme de différenciation, la synthèse d'acide hyaluronique chute tandis qu'une activité hyaluronidase se met en place, résultant en une dégradation du polymère. In vivo, la diffusion de l'acide hyaluronique, comme tout autre composé aqueux au travers de l'épiderme, est bloquée par la barrière lipidique produite dans le stratum granulosum. Les couches inférieures de l'épiderme, riches en acide hyaluronique, drainent les liquides extracellulaires du derme richement hydraté et cette eau ne peut s'échapper au travers des couches supérieures. Le compartiment dermique et les couches épidermiques inférieures constituent donc la réserve d'hydratation de la peau.

La quantité d'acide hyaluronique dans le derme est très largement supérieure à celle contenue dans l'épiderme et représente environ $50 \%$ de l'acide hyaluronique total de l'organisme. Le derme papillaire est plus riche que le compartiment réticulaire indiquant que le fibroblaste du derme papillaire a une capacité de synthèse de l'acide hyaluronique élevée, similaire à celle des fibroblastes synoviaux. Un des protéoglycannes que l'on trouve majoritairement associé à l'acide hyaluronique dans le derme est le versican. Cet hyalectan est capable de se fixer à la fois à l'acide hyaluronique par son domaine $\mathrm{N}$-terminal et aux microfibrilles, des fibres élastiques, la fibrilline 1 et les fibulines 1 et 2 . De ce fait, l'acide hyaluronique est co-distribué avec les fibres élastiques dans le derme.

Bien que la majorité de l'acide hyaluronique soit synthétisée par les fibroblastes dermiques, les cellules épidermiques en sont également capables comme décrit plus haut. Il est cependant intéressant de noter que pratiquement l'entièreté de l'acide hyaluronique a disparu de l'épiderme dans la peau sénile alors qu'il persiste dans le derme âgé suggérant que la régulation de son homéostasie dépend de mécanismes différents dans le derme et l'épiderme. Cependant, si le niveau total d'acide hyaluronique dans le derme reste relativement constant avec l'âge, sa qualité se modifie. La taille du polymère se réduit et il devient moins extractible, suggérant une plus forte association avec les structures tissulaires et peut-être avec un autre répertoire d'hyaladhérines. Ces altérations qualitatives pourraient être responsables de la perte d'hydratation observée dans la peau sénescente [14]. Par ailleurs, si une exposition courte aux UV induit transitoirement un dépôt accru d'acide hyaluronique et une légère réaction œdémateuse, l'exposition répétée aux UV 
déclenche une réponse de type cicatriciel. Les glycosaminoglycannes trouvés dans la peau photo-vieillie sont similaires à ceux présents dans le tissu cicatriciel, avec une proportion réduite d'acide hyaluronique en faveur de protéoglycannes riches en chondroïtine sulfate. Les radicaux libres générés par les UV-B pourraient détruire les polymères d'acide hyaluronique et générer des fragments biologiquement actifs, pro-inflammatoires et pro-angiogènes. Le niveau de synthèse de l'acide hyaluronique est maintenant facilement monitorisé en mesurant l'expression des gènes des HAS présentes dans la peau. Leur expression est stimulée par le TGF $\beta$ aussi bien dans le derme que dans l'épiderme, mais avec des cinétiques différentes. D'autres facteurs de croissance, tel le PDGF, ont également une activité stimulante. Par contre, l'expression des HAS, et donc la production d'acide hyaluronique, est pratiquement complètement éteinte par les glucocorticoïdes. Le dépôt de l'acide hyaluronique dans la matrice extracellulaire et son association avec les hyalectans résultant de l'équilibre entre sa synthèse et sa dégradation, il serait dès lors des plus intéressant d'approfondir l'étude de la biologie des hyaluronidases et des exoglycosidases dans la peau. Il a été évoqué précédemment que dans certaines circonstances, de stress ou de choc, l'organisme était capable de fournir immédiatement de grosses quantités d'acide hyaluronique dans le cadre d'un mécanisme de survie. L'inhibition de la dégradation est un mécanisme nettement plus efficace que l'activation de la synthèse et fait appel à des inhibiteurs endogènes des hyaluronidases. Ces enzymes constituent donc des cibles pharmacologiques potentielles d'intérêt pour contrôler le renouvellement de l'acide hyaluronique dans la peau. Des inhibiteurs exogènes ont déjà été décrits tels que les flavonoïdes, divers actifs végétaux, l'indométhacine, les salicylates, l’héparine.

\section{En conclusion}

Les fonctions biologiques de l'acide hyaluronique dépassent de loin l'image primitive de ce polymère. Par le très large domaine hydrodynamique qu'il occupe, il influence de façon essentielle l'hydratation et les propriétés physiques des tissus. Les interactions qu'il entretient avec d'autres protéines de la matrice extracellulaire en font un acteur primordial dans l'élaboration et le maintien de l'architecture des tissus et de leurs propriétés mécaniques. Enfin, l'acide hyaluronique se lie à des récepteurs à la surface cellulaire pour activer diverses voies de signalisation et influencer le comportement cellulaire. Son maintien dans l'environnement péricellulaire fournit une atmosphère unique à la cellule, la soustrayant aux stimuli extracellulaires, et affecte de façon notable son phénotype, ses mouvements et sa motilité. Ses produits de dégradation acquièrent des propriétés biologiques qui leur sont propres et parfois délétères. Accroître la concentration et la stabilité de l'acide hyaluronique dans la peau, en préserver la taille optimale et induire l'expression du meilleur profil de ses partenaires devraient concourir à maintenir, et peut-être améliorer, l'hydratation cutanée en vue de contrecarrer les marques du temps.

\section{Conflits d'intérêts}

Aucun.

\section{Références}

[1] Itano N. Simple primary structure, complex turnover regulation and multiple roles of hyaluronan. J Biochem 2008;144:131-7.

[2] http: //glycoforum.gr.jp/ cliquer sur «hyaluronan today ».

[3] Meyer J, Palmer JW. The polysaccharide of the vitreous humor. J Biol Chem 1934;107:629-34.

[4] Laurent TC, Fraser JR. Hyaluronan. FASEB J 1992;6:2397-404.

[5] Toole BP. Hyaluronan in morphogenesis. Cell Develop Biol 2001;12:79-87.

[6] Weigel PH, Hascall VC, Tammi M. Hyaluronan synthase. J Biol Chem 1997;272:13997-4000.

[7] Lee JY, Spicer AP. Hyaluronan: a multifunctional, megaDalton, stealth molecule. Curr Opinion Cell Biol 2000;12:581-6.

[8] Puré E, Assoian RK. Rheostatic signaling by CD44 and hyaluronan. Cell Signaling 2009;21:651-5.

[9] Jackson DG. Immunological functions of hyaluronan and its receptors in the lymphatics. Immunol Rev 2009;230:216-31.

[10] Evanko SP, Tammi MI, Tammi RH, Wight TN. Hyaluronandependent pericellular matrix. Adv Drug Delivery Rev 2007;59:1351-65.

[11] Tammi RH, Tammi MI. Hyaluronan accumulation in wounded epidermis: a mediator of keratinocytes activation. J Invest Dermatol 2009;129:1858-60.

[12] Girish KS, Kemparaju K. The magic glue hyaluronan and its eraser hyaluronidase: a biological overview. Life Sciences 2007;80:1921-43.

[13] Stern R, Asari AA, Sugahara KN. Hyaluronan fragments: an information-rich system. Eur J Cell Biol 2006;85:699-715.

[14] Stern R, Maibach HI. Hyaluronan in skin: aspects of aging and its pharmacological modulation. Clin Dermatol 2008;26:106-22. 